\title{
Stokes' and Lamb's viscous drag laws
}

\author{
I. Eames \& C.A. Klettner
}

University College London, Torrington Place, London, WC1E 6BT, U.K.

\begin{abstract}
.
Since Galileo used his pulse to measure the time period of a swinging chandelier in the 17th century, pendulums have fascinated scientists. It was not until Stokes' (1851) (whose interest was spurred by the pendulur time pieces of the mid 19th century) treatise on viscous flow that a theoretical framework for the drag on a sphere at low Reynolds number was laid down. Since then, Stokes famous drag law has been used to determine two fundamental physical constants the charge on an electron and Avogadro's constant - and has been used in theories which have won three Nobel prizes.

Considering its illustrious history it is then not surprising that the flow past a sphere and its two-dimensional analog, the flow past a cylinder, form the starting point of teaching flow past a rigid body in undergraduate level fluid mechanics courses. Usually starting with the twodimensional potential flow past a cylinder, students progress to the three-dimensional potential flow past a sphere. However, when the viscous flow past rigid bodies is taught, the threedimensional example of a sphere is first introduced, and followed by (but not often), the twodimensional viscous flow past a cylinder. The reason why viscous flow past a cylinder is generally not taught is because it is usually explained from an asymptotic analysis perspective. In fact, this added mathematical complexity is why the drag on a cylinder was only solved in 1911, 60 years after the drag on a sphere. In this note, we show that the viscous flow past a cylinder can be explained without the need to introduce any asymptotic analysis while still capturing all the physical insight of this classic fluid mechanics problem.
\end{abstract}




\section{Introduction}

A core theme of fluid mechanics is to understand how impermeable and rigid surfaces such as pipes and particles affect the flow of a fluid. In most undergraduate programmes, fluid mechanics is taught in a sequence that maps onto a student's mathematical maturity. As the breadth and depth of the mathematical skills increases, more detailed problems can be solved and new concepts introduced. When we look at the progression of a student's learning experience in relation to the flow past rigid bodies, all lecturers are met with an identical challenge. At an introductory level, potential flow theory is taught with the focus first being on a two-dimensional cylinder - the simplest and most common geometry - and then the discussion moves onto threedimensional examples, such as a sphere. In an engineering department, for instance, studying the flow past a cylinder is often a prelude to analysing lift on an aerofoil using conformal mapping techniques. Significantly, when we move on to discuss viscous flows past rigid bodies, we always start with the viscous flow past a three-dimensional body (usually a sphere) and rarely, if at all, touch on the viscous flow past a two-dimensional body (usually a cylinder). This is because the usual approach of describing viscous flow past a cylinder draws on asymptotic analysis which is introduced much later in most syllabuses - usually in the third or fourth year - if at all.

The aim of this note is to show the leading order viscous flow past a cylinder can be derived without a rigorous asymptotic analysis and can be introduced at the same time as the viscous flow past a sphere while still providing insight into the drag force experienced by the body and the wake that is generated. Few fluid mechanical results have had such an impact on twentiethcentury theoretical physics than Stokes viscous drag law. To understand the timeliness of Stokes result, it is useful to provide a brief overview of its rich history.

Stokes (1851) paper was largely concerned with the forces on a pendulum, what he describes as the most studied scientific piece of equipment of the age. This is not surprising since pendulums and their related time pieces had been investigated since the early 17th century, by some of the most prominent scientists of their day (Galileo and Huygens to name a few). By the time of Stokes, the obsession for accurate time keeping had moved onto much grander topics such as being able to accurately determine a ships longitude. However the pendulum clock was still a necessary fixture in any upper-middle class Victorian home as reflected in the works of Charles Dickens and Arthur Conan Doyle.

When Stokes published his paper, the subject of fluid mechanics was still in its infancy. Previous force models did not invoke a spatial variation of the velocity field and were heavily influenced by Newton's 'molecular' model. The influence of tangential slip was noted by Sir James South, in a personal communication to Stokes, who observed the relative motion of a gold leaf suspended to a moving pendulum. This observation forced Stokes to account for shear between adjacent layers in the fluid velocity field. Stokes initially laboured with trying to solve the problem of the drag on a long cylinder, failed and moved on to solve the drag on a sphere, using the new method of electrical images. Stokes derived the flowfield based on the neglect of the inertia term and so the governing equation for vorticity is

$$
\nabla^{2} \boldsymbol{\omega}=\mathbf{0} .
$$

Evaluating the force led to a drag law with one disposable coefficient which he proposed to call the index of friction (we now call it the kinematic viscosity). The drag force, $F_{D}$, acting on a rigid sphere of radius $a$ moving with speed $U_{\infty}$ is

$$
F_{D}=6 \pi \mu a U_{\infty},
$$

or the dimensionless measure, the drag coefficient, defined as

$$
C_{D}=\frac{F_{D}}{\frac{1}{2} \rho U_{\infty}^{2} \pi a^{2}}=\frac{24}{R e},
$$


where the Reynolds number, Re, is defined as $R e=2 a U_{\infty} / \nu, \rho$ is the fluid density and $\nu$ is the kinematic viscosity.

Stokes (1851) discussed in detail the failure of his viscous model to determine the force that acts on a cylinder. The usual way to describe this failure is to solve this problem in terms of a scaling of inertia terms, but it is much simpler than this. From (1) we see that $|\tilde{\omega}| \sim \sin \theta / \tilde{r}^{n}$ where $n=1,2$ in two- and three-dimensions respectively. The corresponding flow perturbation scales as $\tilde{\omega} r \sim 1 / \tilde{r}^{n-1}$. Thus the perturbation does not decay in two-dimensions and so a correction to (1) is required to close the problem; this is the Oseen (1910) correction, whose inclusion represents the mathematical challenge. The main difference then between twoand three-dimensions is the requirement to include an Oseen correction. The two-dimensional solution was published 60 years later by Lamb (1911)!

Stokes' result is significant in that it has contributed to three Nobel Prizes in Physics, where the movement of spherical droplets and particles were used as 'probes' in the flow. Robert Millikan developed an oil drop test (around 1909) to measure the electric charge on a droplet and showed that charge is quantised. He was awarded a Nobel Prize in Physics in 1923. The essential feature of Millikan's experiments is that charged oil drops were permitted to fall between two plates across which there was an applied electric field. The charged droplet was created from the breakup of spray break or by introducing an ionising agent such as radium. The experiment consisted of careful measurements of the droplets fall velocity with $\left(v_{2}\right)$ and without $\left(v_{1}\right)$ an electric field (of strength $E$ ) being applied. For steady droplet motion, the balance between electric force, gravity and Stokes' drag law (2) gives

$$
\begin{aligned}
0 & =m g-6 \pi \mu a v_{1}, \\
E e_{n} & =m g-6 \pi \mu a v_{2} .
\end{aligned}
$$

Using the fact that the mass of the droplet, $m=\left(4 \pi a^{3} / 3\right)(\sigma-\rho)$, where $\sigma$ and $\rho$ are the density of the droplet and air respectively and $a$ is the droplet radius, the charge on the droplet could be determined from

$$
e_{n}=\frac{4}{3} \pi\left(\frac{9 \mu}{2}\right)^{3 / 2}\left(\frac{1}{g(\sigma-\rho)}\right)^{1 / 2} \frac{\left(v_{1}+v_{2}\right) v_{1}^{1 / 2}}{E} .
$$

By looking at over two thousand droplets, Millikan was able to show that $e_{n}$ was quantised and the charge on an electron was determined to be

$$
e_{1}=-1.592 \times 10^{-19} C .
$$

The level of care in Millikan's experiments and the corrections considered were extraordinary (see Millikan 1911, 1913).

Brownian motion is another physics problem connected with the drag force on small particles. Robert Brown noted in 1827 the random movement of pollen in alcohol on a slide. To confirm the hypothesis that the animated pollen was not due to swimming, he showed that it also occured with dust. Einstein (1905) published a theory (at the same time as Sutherland 1905) giving an estimate of the macroscopic movement; Einstein's Nobel Prize for Physics in 1921 was for services to theoretical physics.

Sutherland's (1905) argument is much more closely connected to the notion of force which we explain in adapted form. Consider a concentration field of spherical solute particles, with a radius $a$ and which move with velocity $\boldsymbol{v}$ through a dilute solution of viscosity $\mu$. The resistance on each particle is $\boldsymbol{F}_{D}=6 \pi \boldsymbol{v} \mu a$. For $N$ molecules of solute per unit volume, the total resistance will be $N \boldsymbol{F}_{D}$ and in the steady state, diffusion will equilibrate the driving force due to an osmotic pressure, $\nabla p=R T \nabla c$, where $c$ is the solute concentration. For a steady state balance

$$
-R T \nabla c=\boldsymbol{F}_{D} N_{A} c,
$$


(where $N=N_{A} c$ ), or

$$
-\frac{R T}{6 \pi \mu a N_{A}} \nabla c=\boldsymbol{v} c .
$$

The balance between the diffusive and advective flux means that the macroscopic diffusion coefficient is

$$
D=\frac{R T}{6 \pi \mu a N_{A}} .
$$

The calculations of Einstein and Sutherland are remarkable because they connect the macroscopic movement of the solute particles through a random walk and connects the macroscopic movement to a critical number. Millikan estimated Avogadro's constant from $N_{A} e_{n}=F$ where $F$ is Faraday's constant. So by studying the microscopic movement of particles or indeed large molecules, Avogadro's constant can be determined to give a value of

$$
N_{A}=6.062 \times 10^{23} \mathrm{~mol}^{-1} .
$$

Perrin (1910) verified Einstein's prediction experimentally and was awarded the Nobel Prize in Physics in 1926 for contributions to the discontinuous nature of matter. At this time, physics was quite interconnected; Millikan also provided the experimental support for Einstein's photoelectric effect.

Sixty years after Stokes failure to solve the viscous flow past a cylinder, Sir Horace Lamb solved the problem. The mathematical problems arise because the flow tends to decrease so slowly that the far field boundary conditions cannot be satisfied. To solve this problem, Oseen introduced a correction term in the momentum equation to ensure that the far field condition was met. Lamb (1911) derived the drag force on a cylinder for $R e \ll 1$ and showed that

$$
C_{D}=\frac{F_{D}}{\frac{1}{2} 2 a \rho U_{\infty}^{2}} \cong \frac{8 \pi}{R e \log (7.4 / R e)} .
$$

While Lamb's analysis cannot be described as an asymptotic analysis, it does invoke the idea of smallness and expansions. The main difference between two and three-dimensions is that the maths is more difficult. Lamb's paper was really borne from a trick (or decomposition) he had identified to derive the Oseen approximation to flow past a sphere more easily, and the latter part of the paper, is an application to a cylinder. From a teaching perspective, few textbooks (including Lamb's (1932) book Hydrodynamics) give a clear description of viscous flow past a cylinder; Batchelor's (1967) description is not as clear as it is on all other subjects, even for seasoned academics! It is hoped that this paper goes some way in helping the approach to teaching viscous flow past rigid bodies.

The paper is structured as follows. The governing equations of motion are presented in $\S 2$ and the solutions to the case of the cylinder and sphere are given in $\S 3$ and $\S 4$ respectively. The structure of the wake is discussed in $\S 5$. A summary is given in $\S 6$.

\section{Mathematical model}

\subsection{Governing equations}

We consider the leading order flow, with far field velocity $U_{\infty}$, past a cylinder or sphere (of radius $a$ ) in the limit of low Reynolds numbers, where $R e=2 a U_{\infty} / \nu$ where $\nu$ is the kinematic viscosity (see figure 1). The aim is to teach the examples of a cylinder and sphere using a common language and concepts, without appealing to asymptotic analysis. The steady form of the momentum equation is

$$
\rho(\boldsymbol{u} \cdot \boldsymbol{\nabla}) \boldsymbol{u}=-\boldsymbol{\nabla} p+\mu \boldsymbol{\nabla}^{2} \boldsymbol{u},
$$




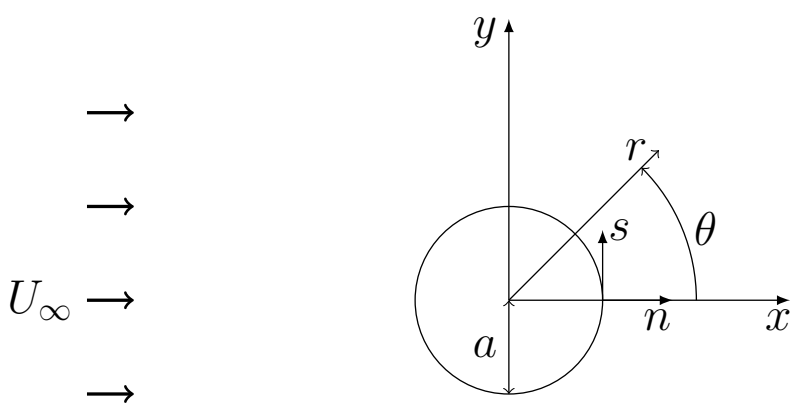

Figure 1: Schematic of the flow past a cylinder or sphere (of radius a) and the coordinate systems used in this work is shown.

where $p$ is the pressure, $\mu$ is the dynamic viscosity and $\rho$ density. The discussion in this paper is based on a non-dimensional form where $\tilde{r}=r / a, \tilde{\boldsymbol{u}}=\boldsymbol{u} / U_{\infty}$ and $\tilde{p}=p / \mu U_{\infty} a$. The vorticity and streamfunction are non-dimensionalised in two- and three-dimensions as

$$
\tilde{\omega}=\frac{a \omega}{U_{\infty}}, \quad \tilde{\psi}=\frac{\psi}{U_{\infty} a},
$$

and

$$
\tilde{\omega}_{\phi}=\frac{a \omega_{\phi}}{U_{\infty}}, \quad \tilde{\Psi}=\frac{\Psi}{U_{\infty} a^{2}},
$$

respectively. On the surface of the body $(\tilde{r}=1)$, the velocity satisfies the no-slip condition

$$
\tilde{u}_{\theta}=\tilde{\boldsymbol{u}} \cdot \hat{\boldsymbol{s}}=0,
$$

and a kinematic condition derived from mass conservation

$$
\tilde{u}_{r}=\tilde{\boldsymbol{u}} \cdot \hat{\boldsymbol{n}}=0,
$$

where $\tilde{u}_{\theta}$ and $\tilde{u}_{r}$ are the tangential and radial components of the velocity vector respectively. $\hat{s}$ and $\hat{\boldsymbol{n}}$ are the unit vectors in the tangential and radial directions respectively. In the far field,

$$
\left(\tilde{u}_{r}, \tilde{u}_{\theta}\right) \rightarrow(\cos \theta,-\sin \theta) .
$$

The boundary conditions $(16,17)$ impose significant constraints on the velocity near the boundary, mainly because $\partial \tilde{u}_{\theta} / \partial \theta=\partial \tilde{u}_{r} / \partial \theta=0$. This means that on the surface of the cylinder, $\partial \tilde{u}_{\theta} /\left.\partial \tilde{r}\right|_{\tilde{r}=1}=\tilde{\omega}_{s}, \partial \tilde{u}_{r} /\left.\partial \tilde{r}\right|_{\tilde{r}=1}=0$. To account for the far field inertia at low Reynolds numbers, the Oseen approximation is applied which uses a linear approximation for the inertia term so that $\tilde{\boldsymbol{u}} \cdot \tilde{\nabla} \tilde{\boldsymbol{u}} \approx \partial \tilde{\boldsymbol{u}} / \partial \tilde{x}$. We seek to determine the leading order solution to

$$
\frac{\partial \tilde{\boldsymbol{u}}}{\partial \tilde{x}}=-\frac{2}{R e} \tilde{\boldsymbol{\nabla}} \tilde{p}+\frac{2}{R e} \tilde{\nabla}^{2} \tilde{\boldsymbol{u}} .
$$

Taking the curl of (19), we see that the vorticity evolves according to

$$
\frac{1}{2} R e \frac{\partial \tilde{\boldsymbol{\omega}}}{\partial \tilde{x}}=\tilde{\nabla}^{2} \tilde{\boldsymbol{\omega}} .
$$

The physical significance of the Oseen term is that it gently sweeps positive and negative vorticity together causing vorticity annihilation and a rapid decrease in $\tilde{\omega}$, this decay is sufficiently fast that a convergent solution can be determined. 


\subsection{Drag coefficient}

The purpose of the calculation is to determine the drag force acting on the body which is defined as

$$
F_{D}=\int_{S_{B}}(p \boldsymbol{I}-\boldsymbol{\tau}) \hat{\boldsymbol{n}} \cdot \hat{\boldsymbol{x}} \mathrm{d} S,
$$

where $\hat{\boldsymbol{n}}$ the unit normal pointing into the cylinder or sphere and $\boldsymbol{\tau}$ ) is the viscous stress tensor. $\hat{\boldsymbol{x}}$ is the unit horizontal vector (see figure 1). The drag coefficients for a cylinder and sphere (see (12) and (3) respectively) can be expressed in terms of the dimensionless surface vorticity, $\tilde{\omega}_{s}$, through

$$
C_{D}=\underbrace{\left.\int_{0}^{2 \pi} \frac{2}{R e} \frac{\partial \tilde{\omega}}{\partial \tilde{r}}\right|_{\tilde{r}=1} \sin \theta \mathrm{d} \theta}_{\text {Pressure component }}-\underbrace{\int_{0}^{2 \pi} \frac{2}{R e} \tilde{\omega}_{s} \sin \theta \mathrm{d} \theta}_{\text {Viscous component }}
$$

and

$$
C_{D}=\underbrace{\int_{0}^{\pi} \frac{4}{R e}\left(\left.\frac{\partial \tilde{\omega}}{\partial \tilde{r}}\right|_{\tilde{r}=1}+\tilde{\omega}_{s}\right) \sin ^{2} \theta \mathrm{d} \theta}_{\text {Pressure component }}-\underbrace{\int_{0}^{\pi} \frac{8}{R e} \tilde{\omega}_{s} \sin ^{2} \theta \mathrm{d} \theta}_{\text {Viscous component }},
$$

respectively (Klettner \& Eames $2016^{1}$ ). These expressions are valid for unsteady flows and all finite Reynolds numbers.

\section{Cylinder}

The non-dimensionalised governing equation with the Oseen correction is shown in Table 2. The derivation for both a cylinder and sphere follow three identical steps which are now explicitly stated here.

Step 1: Transform the Oseen term to give a Helmholtz equation and determine $\tilde{\omega}$.

Our starting point is similar to Lamb (1911) and involves expressing the vorticity field as

$$
\tilde{\omega}=P \exp \left(\frac{R e}{4} \tilde{r} \cos \theta\right) .
$$

This form is chosen to transform the advection-diffusion equation (Table 2) into a Helmholtz equation which can be solved using standard solution techniques. Substituting (24) into the vorticity equation in table 2 gives

$$
\left(\frac{R e}{4}\right)^{2} P=\tilde{\nabla}^{2} P
$$

At low Reynolds numbers, the flow is to leading order symmetric and we seek a solution of the form

$$
P=P_{1}(\tilde{r}) \sin \theta
$$

where from (25), $P_{1}$ satisfies

$$
P_{1}^{\prime \prime}+\frac{P_{1}^{\prime}}{\tilde{r}}-\left(\left(\frac{R e}{4}\right)^{2}+\frac{1}{\tilde{r}^{2}}\right) P_{1}=0 .
$$

The solution to (27) that satisfies $P_{1} \rightarrow 0$ as $\tilde{r} \rightarrow \infty$ is

$$
P_{1}=C_{1} K_{1}(\operatorname{Re} \tilde{r} / 4)
$$

1 The current non-dimensionalisation for $\tilde{\omega}$ was chosen to be consistent with the work of Stokes (1851) and so differs by a factor of two from Klettner \& Eames (2016). 


\begin{tabular}{|c|cc|ccc|}
\hline & $u_{r}$ & $u_{\theta}$ & Kinematic & No-slip & Far field \\
\hline cylinder & $\frac{1}{r} \frac{\partial \psi}{\partial \theta}$ & $-\frac{\partial \psi}{\partial r}$ & $f_{1}(1)=-1$ & $f_{1}^{\prime}(1)=-1$ & $\frac{\mathrm{d} f_{1}}{\mathrm{~d} r} \rightarrow 0$ \\
sphere & $\frac{1}{r^{2} \sin \theta} \frac{\partial \Psi}{\partial \theta}$ & $-\frac{1}{r \sin \theta} \frac{\partial \Psi}{\partial r}$ & $\frac{\partial \tilde{\Psi}}{\partial \theta}=-\cos \theta$ & $\frac{\partial \tilde{\Psi}}{\partial \tilde{r}}=\sin \theta$ & $\tilde{\Psi} \rightarrow 0$ \\
\hline
\end{tabular}

Table 1: Summary of velocity-streamfunction relations where $\tilde{\psi}$ is the streamfunction and $\tilde{\Psi}$ is the Stokes streamfunction and implications for (29) and (42).

\begin{tabular}{|c|c|c|}
\hline \multirow{4}{*}{ cylinder } & Vorticity Equation & $\tilde{\omega}-\tilde{\psi}, \tilde{\omega}_{\phi}-\tilde{\Psi}$ Relationship \\
sphere & $\frac{R e}{2} \frac{\partial \tilde{\omega}}{\partial \tilde{x}}=\tilde{\nabla}^{2} \tilde{\omega}$ & $\tilde{\omega}=-\frac{\partial^{2} \tilde{\psi}}{\partial \tilde{r}^{2}}-\frac{1}{\tilde{r}} \frac{\partial \tilde{\psi}}{\partial \tilde{r}}-\frac{1}{\tilde{r}^{2}} \frac{\partial^{2} \tilde{\psi}}{\partial \theta^{2}}$ \\
\hline$\tilde{x}$ & $\tilde{\nabla}^{2} \tilde{\omega}_{\phi}-\frac{\tilde{\omega}_{\phi}}{\tilde{r}^{2} \sin ^{2} \theta}$ & $\tilde{\omega}_{\phi}=-\frac{1}{\tilde{r} \sin \theta}\left(\frac{\partial^{2} \tilde{\Psi}}{\partial \tilde{r}^{2}}+\frac{1}{\tilde{r}^{2}} \frac{\partial^{2} \tilde{\Psi}}{\partial \theta^{2}}-\frac{1}{\tilde{r}^{2}} \cot \theta \frac{\partial \tilde{\Psi}}{\partial \theta}\right)$ \\
\hline
\end{tabular}

Table 2: Summary of vorticity-streamfunction relations where $\tilde{\psi}$ is the streamfunction and $\tilde{\Psi}$ is the Stokes streamfunction.

where $K_{1}$ is the modified Bessel function of the second kind.

The streamfunction, $\tilde{\psi}$, can be constructed by writing it as the sum of the known free stream component, together with a component to be determined. As such we write,

$$
\tilde{\psi}=\underbrace{\tilde{r} \sin \theta}_{\text {uniform flow }}+\underbrace{f_{1}(\tilde{r}) \sin \theta}_{\text {perturbation }} \text {. }
$$

By substituting (29) into the vorticity-streamfunction relationship (given in Table 2) and considering the lowest order symmetric solution (i.e. $\tilde{\omega} \cong P_{1} \sin \theta$ ) gives

$$
\frac{\mathrm{d}^{2} f_{1}}{\mathrm{~d} \tilde{r}^{2}}+\frac{1}{\tilde{r}} \frac{\mathrm{d} f}{\mathrm{~d} \tilde{r}}-\frac{f_{1}}{\tilde{r}^{2}}=-C_{1} K_{1}(R e \tilde{r} / 4)
$$

Step 2: Solving 2nd order inhomogeneous linear ODE.

Equation (30) is a standard ODE solved subject to the boundary condition in Table 1. This can be solved by writing $f_{1}=\tilde{r} g_{1}$ to give $\tilde{r}^{3} g^{\prime \prime}+3 \tilde{r}^{2} g^{\prime}=-\tilde{r}^{2} C_{1} K_{1}(R e \tilde{r} / 4)$. Integrating

$$
f_{1}=\underbrace{\frac{1}{2}\left(f_{1}^{\prime}(1)+f_{1}(1)-C_{1} \int_{1}^{\infty} \tilde{r} K_{1} \mathrm{~d} \tilde{r}\right) \tilde{r}}_{\text {leading term }}-\frac{1}{2 \tilde{r}}\left(f_{1}^{\prime}(1)-f_{1}(1)-C_{1} \int_{1}^{\infty} \tilde{r}_{1}^{2} K_{1} \mathrm{~d} \tilde{r}\right) .
$$

The far field condition closes the problem and enables $C_{1}$ to be determined

$$
C_{1}=\frac{2}{-\int_{1}^{\infty} K_{1}(\operatorname{Re} \tilde{r} / 4) \mathrm{d} \tilde{r}} \cong \frac{R e}{2 K_{0}(R e / 4)} .
$$


Step 3: Drag coefficient calculation.

From (22),

$$
C_{D}=\frac{2 \pi C_{1}}{R e}\left(\frac{\mathrm{d} K_{1}}{\mathrm{~d} z}-K_{1}\right) .
$$

Using the expansion of $K_{1}$ (see Appendix A, A2) we find the drag coefficient is approximately

$$
C_{D} \cong \frac{8 \pi}{R e \log (7.4 / R e)}
$$

We can see both pressure and viscous stresses contribute equally to the drag force.

\section{Sphere}

The non-dimensionalised equation for the azimuthal vorticity with the Oseen correction for the azimuthal vorticity is shown in Table 2 .

Step 1: Transform the Oseen term to give a Helmholtz equation and determine $\tilde{\omega}_{\phi}$.

Again, our starting point is similar to Lamb (1911) and involves expressing the vorticity field as

$$
\tilde{\omega}_{\phi}=P \exp \left(\frac{R e}{4} \tilde{r} \cos \theta\right) .
$$

This form is chosen to transform the advection-diffusion equation into a Helmholtz equation which has standard solution techniques. Substituting into Table 2 gives

$$
\left(\frac{R e}{4}\right)^{2} P=\tilde{\nabla}^{2} P-\frac{P}{\tilde{r}^{2} \sin ^{2} \theta}
$$

where

$$
\tilde{\nabla}^{2}=\frac{1}{\tilde{r}^{2}} \frac{\partial}{\partial \tilde{r}}\left(\tilde{r}^{2} \frac{\partial}{\partial \tilde{r}}\right)+\frac{1}{\tilde{r}^{2} \sin \theta} \frac{\partial}{\partial \theta}\left(\sin \theta \frac{\partial}{\partial \theta}\right)
$$

Step 2: Solving 2nd order inhomogeneous linear ODE.

At low Reynolds numbers, the local flow is to leading order symmetric and we seek a solution of the form

$$
P=P_{1}(\tilde{r}) \sin \theta
$$

where $P_{1}$ satisfies

$$
\frac{\mathrm{d}^{2} P_{1}}{\mathrm{~d} \tilde{r}^{2}}+\frac{2}{\tilde{r}} \frac{\mathrm{d} P_{1}}{\mathrm{~d} \tilde{r}}-\left(\left(\frac{\tilde{r} R e}{4}\right)^{2}+\frac{2}{\tilde{r}^{2}}\right) P_{1}=0 .
$$

The solution to (39) that satisfies $P_{1} \rightarrow 0$ as $\tilde{r} \rightarrow \infty$ is

$$
P_{1}=C_{2} \frac{1}{\tilde{r}^{2}}\left(1+\frac{1}{4} \tilde{r} R e\right) \exp \left(-\frac{1}{4} R e \tilde{r}\right)
$$

This gives, in Lamb's parlance, the (doubled) angular velocity of the fluid elements (or vorticity)

$$
\tilde{\omega}_{\phi}=C_{2} \frac{\sin \theta}{\tilde{r}^{2}}\left(1+\frac{1}{4} \tilde{r} R e\right) \exp \left(-\frac{1}{4} \operatorname{Re} \tilde{r}(1-\cos \theta)\right)
$$



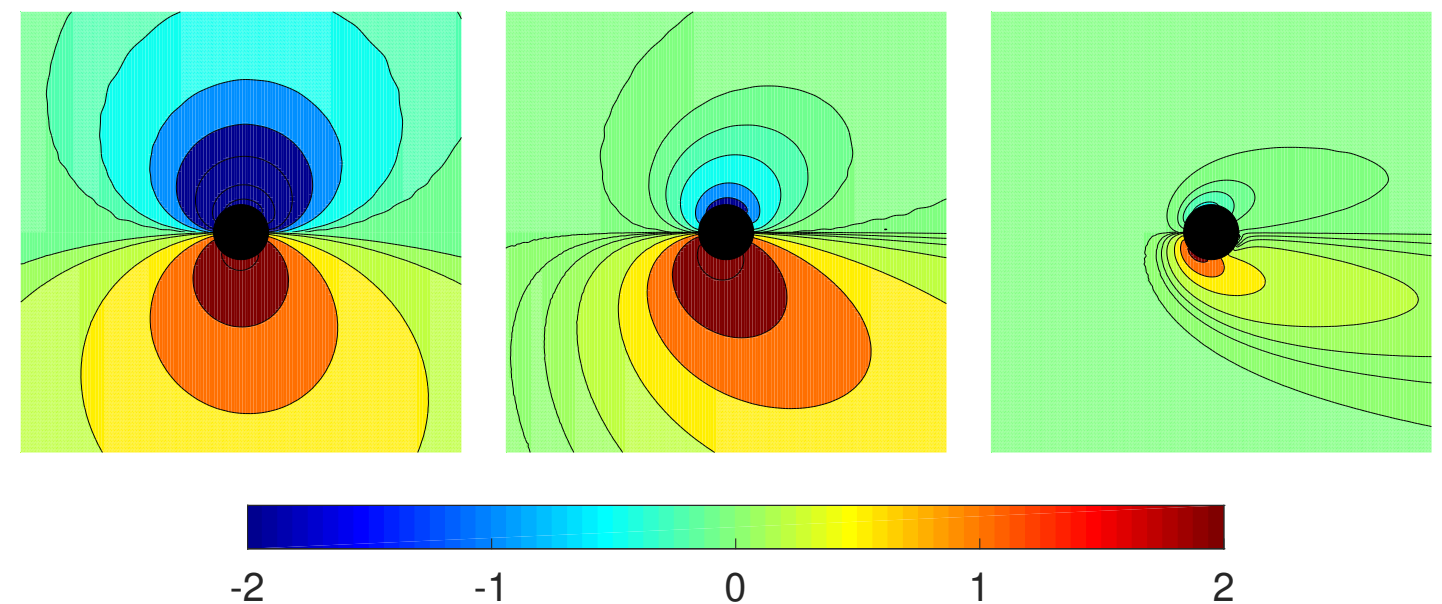

Figure 2: Filled contour distribution of the vorticity field in the vicinity of a sphere (top half) and cylinder (bottom half) are shown for $R e=0.1,1$ and 10 (with the Reynolds number increasing from left to right). The body is at the origin with the flow moving left to right. Here the vorticity field is normalised as $\tilde{\omega} / R e$.

where $C_{2}$ is a constant to be determined.

The Stokes streamfunction, $\tilde{\Psi}$, can be constructed by writing it as the sum of the known free stream component, together with a component to be determined

$$
\tilde{\Psi}=\underbrace{\frac{1}{2} \tilde{r}^{2} \sin ^{2} \theta}_{\text {uniform flow }}+\underbrace{\hat{\Psi}_{1}}_{\text {perturbation }} .
$$

Similar to the cylinder case, substituting (42) into the vorticity-streamfunction relationship (given in Table 2 ) and considering the lowest order symmetric solution (i.e. $\tilde{\omega} \cong P_{1} \sin \theta$ ) gives

$$
\hat{\Psi}_{1}=\frac{1}{4 \tilde{r}} \sin ^{2} \theta-\frac{3}{\tilde{r} R e} \frac{1+\cos \theta}{\sin \theta}\left(1-\exp \left(-\frac{1}{4} \tilde{r} R e(1-\cos \theta)\right)\right) .
$$

The boundary conditions are approximately satisfied to $O(R e)$ on the surface of the sphere with $C_{2}=-3 / 2$.

Step 3: Drag coefficient calculation.

To leading order the vorticity field is $\tilde{\omega}_{\phi}=C_{2} \sin \theta / \tilde{r}^{2}$, so that, from (23)

$$
C_{D}=-\frac{16 C_{2}}{R e}=\frac{24}{R e} .
$$

The ratio of the viscous drag component to the total drag force is quickly obtained and is $2 / 3$.

\section{Far field wake}

The physical consequence of including advection is that it gives an asymmetry to the flow, sweeping the positive and negative vorticity together, causing vorticity annihilation and a much 
faster decay that $1 / \tilde{r}$ and so the solution is determinate. Far downstream, where $\tilde{x} \gg \tilde{y} \gg 1$, the form of the vorticity field behind a cylinder can be calculated from (28) and (A2) to give

$$
\tilde{\omega} \sim C_{1} \sin \theta\left(\frac{2 \pi}{\tilde{r} R e}\right)^{\frac{1}{2}} \exp \left(-\frac{R e}{4} \tilde{r}(1-\cos \theta)\right) \sim C_{1} \frac{\tilde{y}}{\tilde{x}^{3 / 2}}\left(\frac{2 \pi}{R e}\right)^{\frac{1}{2}} \exp \left(-\frac{R e \tilde{y}^{2}}{8 \tilde{x}}\right) .
$$

This means that the vorticity (and velocity) field has a Gaussian profile, whose width scales as $(8 \tilde{x} / R e)^{\frac{1}{2}}$ which can be derived as similarity solution to the linearised vorticity equation in Table 2. The vorticity maximum decreases as $\sim \tilde{x}^{-1}$ due to the positive and negative components diffusing into one another. Likewise, from (41), we can derive the azimuthal vorticity far downstream of a sphere

$$
\tilde{\omega}_{\phi} \sim C_{2} \frac{\tilde{y} R e}{4 \tilde{x}^{2}} \exp \left(-\frac{R e \tilde{y}^{2}}{8 \tilde{x}}\right) .
$$

This shows that the wake flow is Gaussian and that the width grows in a similar manner as the cylinder (with the width scaling as $\left.(8 \tilde{x} / R e)^{\frac{1}{2}}\right)$, but the decay of the maximum vorticity magnitude is faster, as $\tilde{x}^{-3 / 2}$. The vorticity field is shown in figure 2 for a cylinder and sphere for $R e=0.1,1,10$. The symmetry in the vorticity field seen for $R e=0.1$ becomes increasingly asymmetric due to the advection present for the case of $R e=1,10$ (see figure 2).

\section{Conclusion}

We have summarised some of the historical elements relating to viscous flows past rigid bodies, specifically the sphere and cylinder. Stokes' result is significant in its own right but also as an indirect tool to probe fundamental problems in physics in the early 20th century. The closure method of Oseen, applied by Lamb, in resolving the force on a cylinder, is important as a technique. Returning back to the original impetus of this paper, which was to explain Lamb's (1911) results in a way accessible to undergraduates. Hopefully we have shown how this result can be taught in a way that does not require a course in asymptotics and can finally be included in undergraduate material. A step-by-step method is introduced to highlight the similarities and differences between the examples of a sphere and cylinder.

Finally, in this century there are still many fundamental problems in biology, in particular understanding cellular processes. It is not surprising and worth noting that fluid mechanical results such as the viscous drag on long, slender cylinders (Batchelor 1970) feature quite heavily for example in understanding the Brownian motion of proteins and DNA strands.

\section{Appendix A: Useful relationships for modified Bessel function $K_{n}$}

We list the recurrent and asymptotic relationships that are used in this paper.

$$
\frac{\mathrm{d} K_{n}}{\mathrm{~d} z}=\frac{n}{z} K_{n}(z)-K_{n+1}(z) .
$$

The expansion for $K_{1}$ is

$$
K_{1}(z)=\left\{\begin{array}{cc}
\frac{1}{z}+\frac{z}{2} \log \left(\frac{z}{2}\right)+\ldots, & z \ll 1, \\
\left(\frac{\pi}{2 z}\right)^{\frac{1}{2}} \exp (-z), & z \gg 1 .
\end{array}\right.
$$

Expansion for $K_{0}$ is

$$
K_{0}(z) \cong-\gamma-\log (z / 2)
$$

where $\gamma \approx 0.577216$.

[1] Batchelor, G.K. 1967 An Introduction to Fluid Dynamics, 1st edn. Cambridge University Press. 
[2] Batchelor, G.K. 1970 Slender-body theory for particles of arbitrary cross-section in Stokes flow. J. Fluid Mech. 44, 419-440.

[3] Einstein, A. 1905 Ueber die von der molekularkinetischen Theorie der Waerme geforderte Bewegung von in ruhenden Fluessigkeiten suspendierten Teilchen. Annalen der Physik. 17, 549-560.

[4] Klettner, C.A., Eames, I., Semsarzadeh, S. \& Nicolle, A. 2016 The effect of a uniform through-surface flow on a cylinder and sphere. J. Fluid Mech. 793, 798-839.

[5] LAMB, H. 1911 On the uniform motion of a sphere through a viscous fluid. Phil. Mag. 21, 112-121.

[6] Lamb, H. 1932 Hydrodynamics. 6th edn. Cambridge University Press.

[7] Millikan, R.A. 1911 The isolation of an ion, a precision instrument of its charge and the correction of Stokes's law. Physical Review. 32, 349-397.

[8] Millikan, R.A. 1913 On the elementary electrical charge and the Avogadro constant. Physical Review. 2, 109-143.

[9] Oseen, C.W. 1910 Ueber die Stokes'sche Formel und ueber einer verwandte Aufgabe in der Hydrodynamik. Ark. f. Mat. Astr. och Fys. 6, no. 29.

[10] Perrin, J. 1910 Brownian motion and molecular reality. (Translated by F. Soddy) Taylor and Francis London.

[11] Stokes, G.G. 1851 On the effect of the internal friction of fluids on the motion of pendulums. Cam. Phil. Soc. 9, 8-106.

[12] Sutherland, W. 1905 A dynamical theory of diffusion for non-electrolytes and the molecular mass of albumin. Phil. Mag. 9, 781-785. 